\title{
THE MINIMUM MODULUS OF INTEGRAL FUNCTIONS OF SMALL ORDER ${ }^{1}$
}

\author{
P. D. BARRY
}

Communicated by R. P. Boas, November 1960

Let $f(z)$ be an integral function and

$$
M(r)=\max _{|z|=r}|f(z)|, \quad m(r)=\min _{|z|=r}|f(z)| .
$$

If $f(z)$ has order 0 , then the $\cos \pi \rho$-theorem $[1$, p. 40] implies that, for any $\epsilon>0$, there is a sequence of $r \rightarrow \infty$ on which

$$
\log m(r)>(1-\epsilon) \log M(r) .
$$

If $\log M(r)=O\left(\log ^{2} r\right) \quad(r \rightarrow \infty)$ then Hayman [2] proved that (1) holds outside a set of finite logarithmic measure. If $f(z)$ has order at most $\rho$ and $C$ is any constant, then Kjellberg [3, p. 20] showed that

$$
\text { lower log-dens } E\{m(r)>C\} \geqq 1-2 \rho,
$$

and that $1-2 \rho$ is best-possible. Surveys of the main results of this kind are given in $[1 ; 3]$.

In this field I have proved a number of results, of which I state the following. For a given function $\psi(r)(r>0)$ I use the notation

$$
\psi_{1}(r)=d \psi(r) / d \log r, \quad \psi_{2}(r)=d^{2} \psi(r) / d \log ^{2} r,
$$

when these derivatives exist.

Theorem 1. Suppose that

$$
\log M(r) \leqq\{1+o(1)\} \psi(r) \quad(r \rightarrow \infty),
$$

that $\psi_{1}(r)=o\{\psi(r)\} \quad(r \rightarrow \infty)$ and that for $r \geqq r_{0}, 1 / \psi_{2}(r)$ is positive, monotonic decreasing, and a convex function of $\log r$. Then if $0<\delta<1$ and $\epsilon>0$ we have

$$
\begin{aligned}
\text { lower log-dens } E\left\{\log \frac{m(r)}{M(r)}>-(1+\epsilon) \delta^{-1}(2-\delta) \frac{1}{2} \pi^{2} \psi_{2}(r)\right\} & \\
& \geqq 1-\delta .
\end{aligned}
$$

We call a function $L(x)$ continuous and positive for $x \geqq 0$, slowly oscillating, if for each fixed $\lambda>0$,

1 This, in the main, is an abstract of a thesis submitted for the degree of Ph.D. in the University of London, under the supervision of Professor W. K. Hayman. 


$$
\lim _{x \rightarrow \infty} L(\lambda x) / L(x)=1
$$

TheOREM 2. If $\psi_{2}(r)$ is slowly oscillating, $\epsilon>0$, and

$$
\log M(r)=o\{\psi(r)\} \quad(r \rightarrow \infty),
$$

then on a sequence of $r \rightarrow \infty$,

$$
\log m(r)>\left\{1-\frac{1}{2}\left(\pi^{2}+\epsilon\right) \psi_{2}(r) / \psi(r)\right\} \log M(r) .
$$

ThEOREM 3. If $\epsilon>0$ and

$$
\log \log M(r)=o\left\{(\log r)^{1 / 2}\right\}
$$

then

$$
\log \text {-dens } E\{\log m(r)>(1-\epsilon) \log M(r)\}=1 .
$$

THEOREM 4. If $f(z)$ is transcendental and has order at most $\rho$, and $C$ is any constant, then

$$
\text { lower } \log \text {-dens } E\{\log m(r)>C \log r\} \geqq 1-2 \rho \text {. }
$$

The proof of Theorem 2 is based on the ideas of Beurling's theorem $[1$, p. 4] as used by Kjellberg [3, p. 15]. The proofs of Theorems 3 and 4 are based on a result of Beurling $[3$, p. 20]. These results are sharp in some respects.

The special case $\psi(r)=\sigma \log ^{2} r$ in Theorem 1 gives the conclusion lower log-dens $E\left\{m(r) / M(r)>\exp \left(-(1+\epsilon) \delta^{-1}(2-\delta) \pi^{2} \sigma\right)\right\} \geqq 1-\delta$.

The following argument in this special case is typical of the ideas used in proving Theorem 1 , although this particular method does not lead to the best constants.

TheOREM 5. If

$$
\lim \sup _{r \rightarrow \infty} \log M(r) / \log ^{2} r \leqq \sigma,
$$

then, when $0<\delta<1$ and $\epsilon>0$,

$$
\text { lower log-dens } E\left\{m(r) / M(r)>\exp \left(-(1+\epsilon) \delta^{-1} 2 \pi^{2} \sigma\right)\right\} \geqq 1-\delta \text {. }
$$

Proof. Suppose $f(0)=1$. Let $n(r)$ be the number of zeros of $f(z)$ in $|z| \leqq r$. Then by a well-known argument, $n(r)<4(\sigma+\epsilon) \log r$, for all $r \geqq r_{0}(\epsilon)$. If $\left\{r_{n}\right\}$ is the sequence of the moduli of the zeros of $f(z)$ arranged in order of increasing magnitude, we have 


$$
\log M(r) / m(r) \leqq \sum_{n=1}^{\infty} \log \frac{1+r / r_{n}}{\left|1-r / r_{n}\right|} \equiv \phi(r), \text { say. }
$$

The operations involved in the following estimation are justified by standard results about Lebesgue integration.

$$
\begin{aligned}
& \int_{0}^{r} \phi(t) t^{-1} d t=\sum_{n=1}^{\infty}\left\{\int_{0}^{r} \log \frac{1+t / r_{n}}{\left|1-t / r_{n}\right|} t^{-1} d t\right\} \\
& =\sum_{r_{n} \leq r}\left\{2 \sum_{m=0}^{\infty}(2 m+1)^{-1}\left\{\int_{0}^{r_{n}}\left(t / r_{n}\right)^{2 m+1} t^{-1} d t+\int_{r_{n}}^{r}\left(r_{n} / t\right)^{2 m+1} t^{-1} d t\right\}\right\} \\
& +\sum_{r_{n}>r}\left\{2 \sum_{m=0}^{\infty}(2 m+1)^{-1} \int_{0}^{r}\left(t / r_{n}\right)^{2 m+1} t^{-1} d t\right\} \\
& =2 \sum_{m=0}^{\infty}(2 m+1)^{-1}\left\{\sum_{r_{n} \leqq r} r_{n}^{-2 m-1} \int_{0}^{r_{n}} t^{2 m} d t+\sum_{r_{n} \leqq r} r_{n}^{2 m+1} \int_{r_{n}}^{r} t^{-2 m-2} d t\right. \\
& \left.+\sum_{r_{n}>r} r_{n}^{-2 m-1} \int_{0}^{r} t^{2 m} d t\right\} \\
& =2 \sum_{m=0}^{\infty}(2 m+1)^{-1}\left\{\sum_{r_{n} \leqq r}(2 m+1)^{-1}\right. \\
& +\sum_{r_{n} \leq r}(2 m+1)^{-1} r_{n}^{2 m+1}\left(r_{n}^{-2 m-1}-r^{-2 m-1}\right) \\
& \left.+\sum_{r_{n}>r}(2 m+1)^{-1}\left(r / r_{n}\right)^{2 m+1}\right\} \\
& =2 \sum_{m=0}^{\infty}(2 m+1)^{-1}\left\{(2 m+1)^{-1} n(r)\right. \\
& +\int_{0}^{r}(2 m+1)^{-1} t^{2 m+1}\left(t^{-2 m-1}-r^{-2 m-1}\right) d n(t) \\
& \left.+\int_{r}^{\infty}(2 m+1)^{-1}(r / t)^{2 m+1} d n(t)\right\} \\
& =2 \sum_{m=0}^{\infty}(2 m+1)^{-1}\left\{r^{-2 m-1} \int_{0}^{r} n(t) t^{2 m} d t+r^{2 m+1} \int_{r}^{\infty} n(t) t^{-2 m-2} d t\right\} \\
& <16(\sigma+\epsilon) \sum_{m=0}^{\infty}(2 m+1)^{-2} \log r, \quad \text { for } r \geqq r_{1}(\epsilon),
\end{aligned}
$$

i.e., 


$$
\int_{0}^{r} \phi(t) t^{-1} d t<2 \pi^{2}(\sigma+\epsilon) \log r, \quad r \geqq r_{1}(\epsilon) .
$$

Thus if, in a certain set $S$,

$$
\phi(t) \geqq \delta^{-1} 2 \pi^{2}(\sigma+\epsilon),
$$

then for $r \geqq r_{1}(\epsilon)$,

$$
\int_{(1, r) \cap s} t^{-1} d t<\delta \log r
$$

so that

$$
\text { upper } \log \text {-dens } S \leqq \delta .
$$

Theorem 5 follows at once from this.

Some of the results extend to subharmonic functions but a difference between sufficiently slowly growing subharmonic and integral functions is noted.

\section{REFERENCES}

1. R. P. Boas, Jr., Entire functions, New York, Academic Press 1954.

2. W. K. Hayman, Slowly growing integral and subharmonic functions, Comm. Math. Helv. vol. 34 (1960) pp. 75-84.

3. B. Kjellberg, On certain integral and harmonic functions, Dissertation, Uppsala, 1948.

University College, Cork, Ireland 